\title{
Technical validation of a multiplex platform to detect thirty mutations in eight genetic diseases prevalent in individuals of Ashkenazi Jewish descent
}

Charles M. Strom, MD, $P h D^{1}$, Richard A. Janeczko, $P h D^{2}$, Ben Anderson, BS ${ }^{1}$, Joy Redman, $M S^{1}$, Franklin Quan, $P h D^{1}$, Arlene Buller, $P h D^{1}$, Matthew J. McGinniss, $P h D^{1}$, and Wei Min Sun, $P h D^{1}$

\begin{abstract}
Purpose: This study determines the analytic accuracy of a Luminex bead-based commercial analyte-specific reagent for the simultaneous analysis of 30 mutations prevalent in Ashkenazi Jews at eight genetic disease loci. Methods: DNA from 20 samples with known abnormal genotypes were run a total of 109 times. DNA from 820 patients with unknown genotypes submitted for Ashkenazi Jewish testing panels were analyzed using our current laboratory techniques. The 820 samples were then stripped of identifiers, coded, and reanalyzed using the Tm Biosciences (Toronto, Canada) Ashkenazi Jewish panel analyte-specific reagent in a blinded fashion. For the controls, comparisons were made with their known genotypes. For the patient samples, the results of the Tm assay were compared with the results of our current assay. For 24 of the 30 mutations, we had genomic DNA controls or detected patients' samples heterozygous for these mutations. Results: There were no discrepant results in the control or patient samples. In the patient samples, 19,680 genotyping reactions were performed without error in both our laboratory-developed single-disease assays and the Tm multiplex assay. Including the controls, 22,296 genotypes were determined without error. Conclusion: The Tm Biosciences Ashkenazi Jewish analyte-specific reagent is capable of performing accurate analyses of 24 different mutations in eight different genes in a single multiplex reaction and can be used with confidence in the clinical molecular genetics laboratory. Genet Med 2005:
\end{abstract}

$7(9): 633-639$.

Key Words: multiplex, Luminex, Ashkenazi, validation

Molecular genetic carrier testing for individuals of Ashkenazi Jewish (AJ) descent is performed in at least 27 different laboratories in the United States. ${ }^{1}$ There is no consensus on a standard AJ screening panel; as many as 10 different disorders are included by at least one laboratory. ${ }^{1}$ In 2004, the American College of Obstetrics and Gynecology (ACOG) published a committee opinion recommending that individuals of Eastern European Jewish (Ashkenazi) ancestry be offered carrier screening for Tay Sachs disease (TSD), Canavan disease (CD), cystic fibrosis (CF), and familial dysautonomia (FD). ${ }^{2}$ Carrier testing is available for at least six additional diseases affecting this population: Gaucher disease (GD), Fanconi anemia (FA) type C, Niemann-Pick disease (NPD), Bloom syndrome (BS), mucolipidosis IV (ML4), maple syrup urine disease (MSUD), and glycogen storage disease type 1 (GSD1). ${ }^{1}$ Despite the

From the ${ }^{1}$ Genetic Testing Center, Quest Diagnostics Nichols Institute, San Juan Capistrano, California; and ${ }^{2} \mathrm{Tm}$ Bioscience Corporation, Toronto, Ontario, Canada.

Charles M. Strom, MD, PhD, Genetic Testing Center, Quest Diagnostics Nichols Institute, 33608 Ortega Highway, San Juan Capistrano, CA 92690.

Received: June 6, 2005.

Accepted: September 9, 2005.

DOI: 10.1097/01.gim.0000187120.93597.16
ACOG recommendations, many clinicians are testing for more than the four disorders recommended in the committee opinion. For that reason, many laboratories, including ours, offer screening panels for $\mathrm{AJ}$ carrier testing.

In our laboratory, a standard AJ panel consists of eight diseases: the four recommended by ACOG (TSD, CF, FD, and $\mathrm{CD}$ ) and four additional disorders (GD, FA, NPD, and BS). We also perform testing for ML4, MSUD, and GSD1. For historical reasons, ML4, MSUD, and GSD1 are not part of the standard panel. ${ }^{3}$ Many clinicians order ML4, MSUD, and GSD1 concomitantly with the AJ panel.

In our laboratory, patient samples are tested for each individual disease separately, even when a complete panel is ordered. Therefore, even though a single tube of blood is submitted for AJ panel testing, eight separate DNA preparations and assay setups are performed on each patient sample. This results in higher costs, longer turnaround times, and increased logistical complications than if these tests were performed simultaneously. A reagent system that would allow simultaneous testing for all AJ diseases and mutations would be a significant advance. This study describes the evaluation of a commercially available analyte-specific reagent (ASR) supplied by Tm Biosciences (Toronto, Canada) capable of the simultaneous genotyping of 30 mutations in eight different genes for AJ diseases. 


\section{METHODS}

\section{DNA purification}

Patient samples were prepared using a Genovision Biorobot (Qiagen, Venlo, The Netherlands) under conditions recommended by the manufacturer. One-hundred microliters of anticoagulated whole blood were placed into 96-well microtiter plates and placed into the Biorobot. The resulting DNA solution was diluted $1: 2$, and $2.5 \mu \mathrm{L}$ were removed for the polymerase chain reaction (PCR). No DNA quantification was performed. For rare mutations in which genomic controls were unavailable, synthetic oligonucleotides were supplied by Tm Biosciences. Control DNA samples were prepared by Gentra (Toronto, Canada) DNA purification robotic stations under conditions specified by the manufacturer. These samples were diluted to a concentration of $10 \mathrm{ng} / \mu \mathrm{L}$, and $2.5 \mu \mathrm{g}$ were used in PCR.

\section{OLIGONUCLEOTIDES}

All oligonucleotides were synthesized on a $250 \mathrm{nmol}$ scale (Integrated DNA Technologies, Coralville, IA). Universal antitags were amino-modified on $3^{\prime}$ terminus to allow for subsequent coupling to carboxylated microspheres (Luminex, Austin, TX). Antitag oligonucleotides were purified by reversephase high-performance liquid chromatography. Unmodified PCR primers were desalted.

\section{Tagged allele-specific primer elongation primers}

Primers and chimeric primers composed of a 24-mer universal tag sequence on the $5^{\prime}$ end and a variable length allelespecific sequence on the $3^{\prime}$ end were unmodified and purified by polyacrylamide gel electrophoresis. After reconstitution in RNase/DNase-free water, all oligonucleotides were quantified by spectroscopy at $260 \mathrm{~nm}$. Oligonucleotide concentrations were determined using the absorbance measurements at 260 $\mathrm{nm}$, and the extinction coefficients were provided by the supplier.

\section{MICROSPHERE COUPLING}

Each of the 86 amino-modified anti-tag sequences was coupled to a corresponding population of carboxylated fluorescent microspheres following a modified carbodiimide coupling procedure as described previously. ${ }^{4}$

\section{MULTIPLEX POLYMERASE CHAIN REACTION}

The mutations detected are shown in Table 1 and were analyzed after an 18-plex PCR reaction in a $12.5-\mu \mathrm{L}$ volume containing $30 \mathrm{mmol} / \mathrm{L}$ Tris- $\mathrm{HCl}, \mathrm{pH} 8.4,75 \mathrm{mmol} / \mathrm{L} \mathrm{KCl}, 2$ $\mathrm{mmol} / \mathrm{L} \mathrm{MgCl}_{2}, 200 \mu \mathrm{mol} / \mathrm{L}$ of each dNTP (Roche, Basel, Switzerland), primers (see below), 25 ng genomic DNA, and 5 units Platinum Taq polymerase (Invitrogen, Mississauga, Ontario, Canada). PCR primer concentrations ranged from 0.128 to $1.28 \mu \mathrm{mol} / \mathrm{L}$ per reaction. Amplification reactions were performed in an MJ Research PTC-100 thermocycler using the following cycling parameters: an initial 5-minute denaturation at $95^{\circ} \mathrm{C}$ followed by 30 cycles of denaturation at $95^{\circ} \mathrm{C}$ for 30 seconds, annealing at $58^{\circ} \mathrm{C}$ for 30 seconds, and extension at $72^{\circ} \mathrm{C}$ for 30 seconds. A final extension step was performed by incubating for 5 minutes at $72^{\circ} \mathrm{C}$. The ramp rate was maintained at $1.5^{\circ} \mathrm{C}$ per second. Products were held at $4^{\circ} \mathrm{C}$ until further use.

\section{Post-polymerase chain reaction processing}

Each $12.5 \mu \mathrm{L}$ of PCR product was treated with 1.25 units of shrimp alkaline phosphatase (USB Corporation, Cleveland, Ohio) and 5 units Exonuclease I (USB Corporation, Cleveland, Ohio). Samples were incubated for 30 minutes at $37^{\circ} \mathrm{C}$, and the enzymes were denatured by incubating for 15 minutes at $99^{\circ} \mathrm{C}$.

\section{MULTIPLEX ALLELE-SPECIFIC PRIMER EXTENSION}

Allele-specific primer extension (ASPE) was performed in a $20-\mu \mathrm{L}$ reaction containing a $5-\mu \mathrm{L}$ aliquot of treated PCR product. Each reaction consisted of $20 \mathrm{mmol} / \mathrm{L}$ Tris- $\mathrm{HCl}, \mathrm{pH} 8.4,50$ $\mathrm{mmol} / \mathrm{L} \mathrm{KCl}, 1.25 \mathrm{mmol} / \mathrm{L} \mathrm{MgCl}_{2}, 4.5$ units of Platinum Tsp polymerase (Invitrogen), $8 \mu \mathrm{mol} / \mathrm{L}$ each of biotin-dCTP, dATP, dGTP, and dTTP (Roche), and 24 nM ASPE primer pool (containing the 61 universally tagged primers). The ASPE reactions were incubated for 2 minutes at $96^{\circ} \mathrm{C}$ and then subjected to 40 cycles for 30 seconds at $95^{\circ} \mathrm{C}, 30$ seconds at $52^{\circ} \mathrm{C}$, and 60 seconds at $74^{\circ} \mathrm{C}$. Reactions were maintained at $4^{\circ} \mathrm{C}$ until further use.

\section{Universal array sorting}

ASPE reaction products were hybridized to a population of Luminex microspheres containing appropriately coupled antitags. Each bead population had a complementary anti-tag (61 bead populations) corresponding to the tag on each allele-specific primer used. Beads (at a concentration of 2500 for each bead) were pooled, centrifuged at 10,000 rpm for 60 seconds, and resuspended in $1.1 \times$ hybridization buffer $(0.22 \mathrm{~mol} / \mathrm{L}$ $\mathrm{NaCl}, 0.11 \mathrm{~mol} / \mathrm{L}$ Tris, $\mathrm{pH} 8.0$, and $0.088 \%$ [v/v] Triton $\mathrm{X}-100)$. A $45-\mu \mathrm{L}$ aliquot of this bead mixture was added to each sample well of a 96-microwell plate (Costar, Corning, New York). A 5- $\mu \mathrm{L}$ aliquot of ASPE product was added directly to the wells for a final hybridization volume of $50 \mu \mathrm{L}$. Samples were heated to $96^{\circ} \mathrm{C}$ for 2 minutes, and hybridization was performed at $37^{\circ} \mathrm{C}$ for 1 hour. Hybridized samples were transferred to a prewetted $1.2 \mu \mathrm{mol} / \mathrm{L}$ Durapore membrane (Millipore, Billirica, Massachusetts) and washed with $200 \mu \mathrm{L}$ wash buffer $(0.2 \mathrm{~mol} / \mathrm{L} \mathrm{NaCl}, 0.1 \mathrm{~mol} / \mathrm{L}$ Tris, $\mathrm{pH} 8.0$, and $0.08 \%$ [v/v] Triton X-100). For detection, $150 \mu \mathrm{L}$ of reporter solution ( $1 \mathrm{mg} / \mathrm{L}$ Streptavidin-R-Phycoerythrin in wash buffer; Molecular Probes Inc., Carlsbad, California) was added to each sample well and incubated for 15 minutes in the dark. The samples were analyzed on the Luminex xMAP instrument at ambient temperature. The instrument was set to read a minimum of 100 events per bead population, maintaining a gate setting established with bead mix before the samples were run. Dual lasers simultaneously identified the color-coded microspheres and fluorescently labeled extension products to generate a signal for each variation. 
Table 1

Testing results on 820 patient samples and 109 control samples for 30 mutations in 8 Ashkenazi Jewish diseases

\begin{tabular}{|c|c|c|c|c|c|}
\hline Disease & Locus & Amplicon Size & Mutation & Number Control & Number Patient \\
\hline \multirow[t]{8}{*}{ Gaucher } & GBA & $1290 \mathrm{bp}$ & 1035insG & 5 & 2 \\
\hline & & 1313 bp & $\operatorname{IVS} 2(+1) \mathrm{G}>\mathrm{A}$ & 5 & 0 \\
\hline & & & N370S & 6 & 26 \\
\hline & & & L444P & 5 & 1 \\
\hline & & & Del55bp ${ }^{a}$ & 0 & 0 \\
\hline & & & $\mathrm{V} 394 \mathrm{~L}^{a}$ & 0 & 1 \\
\hline & & & $\mathrm{D} 409 \mathrm{H}^{a}$ & 0 & 0 \\
\hline & & & $\mathrm{R} 496 \mathrm{H}^{a}$ & 0 & 2 \\
\hline \multirow[t]{2}{*}{ Fanconi Anemia } & FANCC & 253 bp & $\operatorname{IVS} 4(+4) \mathrm{A}>\mathrm{T}$ & 5 & 9 \\
\hline & & 273 bp & $322 \mathrm{delG}^{a}$ & 0 & 0 \\
\hline \multirow[t]{2}{*}{ Familial Dysautonomia } & IKBKAP & 600 bp & $2507+6 \mathrm{~T}>\mathrm{C}$ & 6 & 19 \\
\hline & & & $\mathrm{R} 696 \mathrm{P}^{a}$ & 0 & 1 \\
\hline \multirow[t]{4}{*}{ Niemann-Pick } & SMPD1 & $331 \mathrm{bp}$ & L302P & 5 & 1 \\
\hline & & 536 bp & fs 330 & 5 & 2 \\
\hline & & & $\mathrm{R} 496 \mathrm{~L}$ & 5 & 5 \\
\hline & & & DelR608 & 5 & 0 \\
\hline \multirow[t]{7}{*}{ Tay Sachs } & HexA & 284 bp & $\mathrm{R} 247 \mathrm{~W}$ & 5 & 2 \\
\hline & & 344 bp & G269S & 5 & 1 \\
\hline & & 411 bp & 1278insTATC & 5 & 17 \\
\hline & & 494 bp & $\operatorname{IVS} 12(+1) \mathrm{G}>\mathrm{C}$ & 5 & 7 \\
\hline & & & $\mathrm{R} 247 \mathrm{~W}^{a}$ & 0 & 0 \\
\hline & & & $\operatorname{IVS} 9(+1) \mathrm{G}>\mathrm{A}^{a}$ & 0 & 0 \\
\hline & & & del7.6kb ${ }^{a}$ & 4 & 0 \\
\hline Bloom Syndrome & RECQ2 & 235 bp & 2281del6/ins7 & 6 & 0 \\
\hline \multirow[t]{4}{*}{ Canavan Disease } & ASPA & $211 \mathrm{bp}$ & $\mathrm{Y} 231 \mathrm{X}$ & 5 & 0 \\
\hline & & 229 bp & E285A & 5 & 9 \\
\hline & & 295 bp & A305E & 5 & 1 \\
\hline & & & $433(-2) \mathrm{A}>\mathrm{G}^{a}$ & 0 & 0 \\
\hline \multirow[t]{2}{*}{ Mucolipidosis IV } & & $363 \mathrm{bp}$ & Del6.4kb & 5 & 2 \\
\hline & & 402 bp & $\operatorname{IVS} 3(-2) A>G$ & 5 & 4 \\
\hline \multicolumn{6}{|l|}{ Total } \\
\hline \multirow[t]{2}{*}{8 Diseases } & & & 20 mutations & 102 & 108 \\
\hline & & & 10 mutations $^{a}$ & 4 & 4 \\
\hline
\end{tabular}

${ }^{a}$ Mutations on the Tm panel but not on the current menu.

bp, base pair. 


\section{DATA ANALYSIS AND INTERPRETATION}

Tag-IT Data Analysis Software was used to analyze the data output file generated by the Luminex (TmBiosciences, Toronto, Canada) instrument. To perform the analysis of a sample, the software used the median fluorescence intensity (MFI) values for each allele of each mutation for that sample, as well as the MFI values of a PCR-negative control sample. The maximum MFI allowed for the PCR-negative control was 200. If any signal on any bead exceeded 200 for the PCR-negative controls, the entire plate of samples was failed and the analysis was stopped. Otherwise, NET MFI values were calculated by subtracting the PCR-negative control MFI values from the respective MFI values of the sample being analyzed; NET MFI values that are calculated to be negative are set to zero. Acceptance criteria were set such that, for each mutation, the MFI value for at least one allele was required to be $10 \times$ the corresponding PCR negative control MFI value and at least $300 \mathrm{MFI}$ units. If these criteria were not met, the sample in question was failed and no mutations were called. If a sample passed, the genotype for each mutation was determined on the basis of allelic ratios (ARs), where the AR is equal to the NET MFI for an allele divided by the sum of the NET MFI for all alleles tested for that mutation. Therefore, for the usual bi-allelic variation there is a wild-type AR and a mutant AR whose sum is 1 by definition. ARs represent the fraction of the total net MFI signal for a given variation attributed to the presence of a particular allele. ARs are used as opposed to net signals because they normalize for variability in signals between loci and between samples. In this study, the AR ranges were set as follows: If the wild-type AR was at least 0.85 , the call was "WT" (only the wild-type allele has been detected); if the AR for both alleles in a bi-alleic system was at least 0.30 , the call was heterozygous for these two alleles ("HET"); if only the AR for a mutant allele was at least 0.30 , the call was "Mu D" (Mutant Detected: the mutant allele was detected; in the case of tri-allelic variations, the detected mutant allele was specified; in all other cases, the call was "No Call” (ambiguous signals).

As with all primer extension-based assays, underlying mutations/polymorphisms occurring in the ASPE primer binding regions may affect the signals generated and consequently the calls made. If hybridization of the wild-type ASPE primer is affected by an underlying mutation/polymorphism, a sample that is heterozygous for a given mutation may appear (based on the AR) to be homozygous mutant. Homozygous wild-type calls accurately reflect the genotype unless two (or more) mutations occur within the ASPE primer hybridization region of the same CFTR gene. Heterozygous calls will accurately reflect the genotype, because underlying mutations cannot give a spurious appearance of heterozygosity.

\section{DNA sequencing}

DNA sequencing was performed using BigDye (Alameda, California) reagent $(\mathrm{ABI})$ and analyzed on $\mathrm{ABI}$ model 3730 automated sequencers as described previously. ${ }^{5}$

\section{Individual assays}

Assays for the eight diseases described in Table 1 were performed exactly as described previously. ${ }^{3}$

\section{RESULTS \\ DNA samples}

A total of 109 samples of genomic DNA prepared from either cell lines purchased from Coriell Mutant Cell Repository (Camden, New Jersey) or from anonymized samples submitted to the laboratory for analysis and found to harbor mutations were analyzed. Samples were placed into open positions on 96-well titer plates following College of American Pathology/Clinical Laboratory Improvement Act regulations. In all, 820 sequential patient samples submitted for AJ panel testing were analyzed. All of the samples were also anonymized and analyzed for ML4 mutations for confirmation of the Tm results. The identity of each sample was blinded to the laboratory.

\section{Mutations analyzed}

The mutations analyzed by our current assay and the Tm ASR are shown in Table 1. For the purposes of clarity, the "common" notation for these mutations is used, rather than any proposed standardized nomenclature. The Tm ASR contains all the mutations currently analyzed in our laboratory plus an additional 10 mutations not currently assayed. At the onset of the study, we did not have genomic controls for 9 of the 10 additional mutations. The French Canadian TSD mutation del6.7kb is on our non-AJ TSD panel, and therefore we had genomic controls DNA for this mutation. The Tm ASR was able to detect the deletion in the genomic control samples. Not unexpectedly, no patients tested positive for this mutation because these samples were submitted for AJ panel screening and this mutation is prevalent in French Canadians. For three of the remaining nine mutations, although no controls were available, patients were detected with those mutations. DNA sequencing was performed to confirm that these patients were indeed heterozygous for these mutations. Therefore, we can confirm the ability of the Tm ASR to detect in genomic DNA 24 of the 30 mutations claimed to be detectable by the manufacturer. Because the samples had been anonymized, these results could not be communicated to the ordering physician.

For the remaining six mutations (del55bp and $\mathrm{D} 409 \mathrm{H}$ in $\mathrm{GD}, 322$ delG in FA, 433[-2]A $>\mathrm{G}$ in $\mathrm{CD}, \mathrm{R} 247 \mathrm{~W}$ and IVS9 $[+1] \mathrm{G}>\mathrm{A}$ in TSD, and $433[-2] \mathrm{A}>\mathrm{G}$ ) no carriers were detected. The ASR reagent was able to detect these mutations using the synthetic controls supplied by the manufacturer. Because no genomic DNA controls were available, we can make no conclusions regarding the Tm assay's ability to accurately detect these mutations on genomic DNA samples. We have had multiple experiences in the past when developing genotyping assays in which mutations in synthetic DNA were detected well but the assay failed to reliably detect the same mutation in a genomic sample. 


\section{Accuracy of genotyping results}

There were no discrepancies between the known genotype and the Tm-determined genotype in the 109 control DNA samples tested. There were eight control DNA samples containing two mutations, and these were also detected flawlessly. There were no discrepancies observed in the 820 sample patient series using our standard assays and the Tm assay. A total of 105 patients were found to have at least one mutation (12\%), and seven patients were compound heterozygotes for two mutations $(0.8 \%)$. These are similar to the frequencies we observed in our previous study. ${ }^{3}$ Therefore, in the patient series, 820 samples were analyzed flawlessly for 24 mutations yielding 19,680 consecutive, accurate genotypes. Adding the control series of 109 analyses yielded a grand total of 22,296 accurate genotyping results.

\section{Robustness of the assay}

In addition to the accuracy of an assay, its robustness is also an important consideration. The Tm ASR performed much better on fresh DNA samples than on DNA samples that had been stored for periods of time ranging from 5 to 7 months. These stored samples required the addition of more DNA to the reaction than fresh DNA samples. Approximately $20 \%$ of control samples required a repeat analysis using more DNA to obtain a successful genotype for all mutations.

In our laboratory procedure, when a sample fails any of the mutation analyses, the blood tube is retrieved and the analysis proceeds from the beginning. If the sample cannot be genotyped for all mutations after three attempts, a result of "failure to genotype" is reported. In this series, with the use of freshly prepared genomic DNA from submitted blood samples, 96\% of samples yielded genotypes on the first attempt for all 24 mutations. This is an acceptable rate for most laboratories for an individual assay and an impressive rate for an assay detecting 24 mutations in eight different diseases. The 33 samples requiring a repeat analysis were all successfully genotyped in the second analysis. In 15 samples, multiple mutations failed, attributable to poor DNA quality or quantity. Two samples failed because of low bead count, probably the result of clumping and loss of beads during processing. In nine samples there was single mutation failure for the IVS 2 mutation in GD. In six samples, other mutations failed in combination with IVS 2. A single sample failed the del6.4 ML4 mutation analysis because the signal was greater than the software cutoff value. In general, the IVS 2 GD mutation, which requires a long amplicon of greater than $1.0 \mathrm{~kb}$, seems to be the most prone to failure. However, only $1 \%$ of failures were the result of an isolated problem with this mutation. We are attempting to adjust PCR conditions to improve the performance of this mutation analysis.

\section{Throughput and automation}

The Tm ASR is amenable to 96-well plate formats and automated processing. All pipetting steps were performed by liquid handling stations. The total processing time from the end of
DNA isolation to placement into the Luminex instrument was approximately 7 hours and 30 minutes. This includes all labor and instrument time. We found a single Luminex instrument to be capable of analyzing approximately 140 samples per hour. There is an analysis time of approximately 25 seconds per sample followed by washing and priming. Thus, all the preanalytic procedures can be completed in a single 8-hour shift and the 96-well plates can be placed on the Luminex instruments, which can analyze the entire plate without human intervention. This is suitable for a high-throughput laboratory.

Software provided by Tm Biosciences was capable of making allele assignments and interfacing with laboratory information systems computers to upload results. In addition, we designed software to determine which tests were ordered for each patient and to analyze and report results only for those tests specifically ordered by the physician. Because the directors reviewing the analysis are not shown any data from tests that were not ordered, compliance issues are avoided such as what to do about a positive result for a test the physician did not order.

\section{DISCUSSION}

We performed a technical validation of an ASR reagent that is capable of performing simultaneous analysis of 30 mutations in eight diseases frequent among individuals of $\mathrm{AJ}$ descent. We currently offer analyses for 48 mutations in 11 diseases. The 8 diseases and 20 mutations are listed in Table 1: CF (23 mutations), GSD1 (2 mutations), and MSUD (3 mutations). Currently, each disease is tested separately, with the exceptions of MSUD and GSD, which are assayed simultaneously. This requires eight separate DNA preparations and eight separate PCR reactions, and eight separate analyses and at least 16 separate reviews (each molecular result is reviewed at least twice before reporting) to complete the analyses. Reagent and labor costs would be reduced dramatically by incorporating this assay into the laboratory. The Tm ASR required a $4 \%$ repeat rate in this series of 820 patients. Therefore, $96 \%$ of samples can be reported after a single round of analysis. In contrast, if each of the eight separate assays we currently perform has even a $2 \%$ repeat rate, theoretically all eight assays will be successfully completed in one round of testing only $85 \%$ of the time. In practice the number is closer to $80 \%$. Having to repeat at least one test causes delays in turnaround time because the entire panel is not reported until all results are available. Because these tests are usually ordered and performed for pregnant women, the ability to report results faster is a significant advance.

The need for developing DNA analytic platforms capable of performing multiplex analysis became evident in the case of $\mathrm{CF}$ carrier detection. Currently, 23 mutations and 5 polymorphisms are required for $\mathrm{CF}$ carrier detection according to recommendations from the American College of Medical Genetics $^{6}$ and the ACOG. ${ }^{7}$ With the explosion of information derived from the completion of the Human Genome Project, molecular diagnostics has an increasingly important role in 
clinical medicine. Only rarely can a single mutation analysis provide the necessary information to thoroughly investigate a genetic disease state.

We previously evaluated four different platforms capable of performing accurate multiplex genotyping, the Roche CF Gold Lipa Strips ASR reagent ${ }^{8}$ (Roche Molecular Diagnostics), Oligonucleotide Ligation Assay (OLA) CF Genotyper ASR reagent, ${ }^{8,9}$ a proprietary DNA chip, ${ }^{9}$ and a Luminex-based CF $40+4$ ASR reagent (Tm Biosciences).

The Lipa system is suitable for laboratories performing lowvolume testing because the technology is not easily amenable to automation for high-throughput laboratories. In addition, the physical limitations of positions on the strip make it difficult to increase the number of mutations detected in a single strip.

The OLA-based assay is robust, automatable for highthroughput testing, and capable of analyzing a sufficient number of mutations. There are two major drawbacks to this technology; analysis requires expensive and sophisticated instruments, namely, automated DNA analyzers that can cost hundreds of thousands dollars and require extensive preventative maintenance, and because there is a patent on OLA technology, it precludes its use in a laboratory developed test or by another in vitro diagnostic company.

Currently, manufacturing DNA chips and optimizing assays is a long process not easily amenable to rapid assay development. The Luminex system currently has 100 colors available, making 50 mutation genotyping possible. It is an "open platform" allowing laboratories or IVD companies to purchase beads for assay development. Multiple chemistries or hybridization-based assays can be performed on the beads. Tm has chosen ASPE, but, in our experience, OLA works equally well and theoretically, any other detection method such as Promega's ReadIT ${ }^{3}$ could be adapted to the Luminex system.

A multiplex assay for more than 30 mutations will likely be necessary to perform newborn screening for primary congenital glaucoma (PCG). PCG is a congenital disorder causing increased intraocular pressure beginning in the newborn period. The incidence in the United States is approximately 1:10,000 births. Infants with PCG are rarely diagnosed until after the eyeball has begun to protrude and visual loss has already occurred. In developed countries, late-diagnosed PCG accounts for approximately $10 \%$ to $20 \%$ of all adult blindness. Currently, there is no reliable method of detecting elevated intraocular pressures in infants without general anesthesia. At least $80 \%$ of PCG is caused by mutations in the cytochrome p4501B1 gene (CYP1B1). There are more than 30 mutations described in CYP1B1 capable of causing PCG in various populations.

An accurate and inexpensive newborn screening test could identify infants who need an examination under anesthesia; if increased ocular pressure is diagnosed, surgery could be performed to reduce the pressure before loss of vision occurs. Because early diagnosis of PCG can enable surgical intervention to prevent the developing visual handicap, this disorder would be an excellent candidate for newborn screening. ${ }^{10-12}$
The investigation of a single genetic disease may require the simultaneous analysis of several mutations in several different genes. Currently, molecular investigation of individuals with disease states such as thrombophilia are most effectively investigated by simultaneous analysis of Factor $\mathrm{V}$ Leiden (p.R506Q), the HR-2 mutation in factor V, the c.20210G >A mutation in the prothrombin (Factor II) gene, and the c. $677 \mathrm{C}>\mathrm{T}$ mutation in methylene tetrahydrofolate reductase. ${ }^{13,14}$

Performing each mutation analysis as a separate assay makes testing costly, time consuming, and logistically complex. In the case of diseases frequent in individuals of AJ ancestry, multiplex testing allows samples to be analyzed for eight diseases and 24 mutations simultaneously, thus drastically decreasing turnaround time and costs, and simplifying the logistics of reporting 11 different results. A single DNA preparation has several advantages. It decreases the amount of blood necessary to complete the testing, the cost of testing because of reagent and labor savings, and the test completion time.

The Luminex platform with its 100 bead color system is theoretically capable of testing for 50 mutations simultaneously. There are no current clinical indications surpassing that number. Even if $\mathrm{CF}$ were combined with the entire 11 disease AJ testing panel, 48 mutations would be analyzed. There are many different chemistries that can be used in tandem with the Luminex-based system.

Because individuals will usually have only a single genotyping assay in their lifetime, it is essential that clinical molecular laboratories choose testing platforms capable of performing highly accurate genotyping. ${ }^{13}$ Because of this mandate, we have instituted a 1000-sample benchmark test before introducing a new test into the laboratory. ${ }^{15}$ The ability to perform 22,296 genotyping assays without error is impressive and allows a clinical laboratory to proceed with confidence when performing multiplex assays using this platform. We expect this and other Luminex-based multiplex assay reagents to revolutionize clinical molecular laboratories.

\section{References}

1. Leib JR, Gollust SE, Hull SC, Wilfond BS. Carrier screening panels for Ashkenazi Jews: is more better? Genet Med 2005;7:185-190.

2. American College of Obstetrics and Gynecology Committee on Genetics. Prenatal and preconceptional carrier screening for genetic diseases in individuals of Eastern European Jewish descent. Obstet Gynecol 2004;104:425-428.

3. Strom CM, Crossley B, Redman JB, Quan F, et al. Molecular screening for diseases frequent in Ashkenazi Jews: lessons learned from more than 100,000 tests performed in a commercial laboratory. Genet Med 2004;6:145-151.

4. Bortolin S, Black M, Modi H, Boszko I, et al. Analytical validation of the tag-it high-throughput microsphere-based universal array genotyping platform: application to the multiplex detection of a panel of thrombophilia-associated single-nucleotide polymorphisms. Clin Chem 2004;50:2028-2036.

5. Strom C, Huang S, Chen C, Buller A, et al. Extensive sequencing of the cystic fibrosis transmembrane regulator gene: Assay validation and unexpected benefits of developing a comprehensive test. Genet Med 2003;5:9-14.

6. Grody WW, Cutting GR, Klinger KW, Richards CS, et al. Laboratory standards and guidelines for population-based cystic fibrosis carrier screening. Genet Med 2001;3: 149-154.

7. American College of Obstetrics and Gynecology and American College of Medical Genetics. Preconception and Prenatal Carrier Screening for Cystic Fibrosis, Clinical 
and Laboratory Guidelines. Washington, DC: American College of Obstetrics and Gynecology; 2001.

8. Strom C, Huang D, Buller A, Redman J, et al. Cystic fibrosis screening using the college panel: platform comparison and lessons learned from the first 20,000 samples. Genet Med 2002;4:289-296.

9. Strom CM, Clark DD, Hantash FM, Maul D, et al. Direct visualization of cystic fibrosis transmembrane regulator mutations in the clinical laboratory setting, Clin Chem 2004;50:836-845.

10. Stoilov I, Akarsu AN, Alozie I, Child A, et al. Sequence analysis and homology modeling suggest that primary congenital glaucoma on 2 p21 results from mutationsdisrupting either the hinge region or the conserved core structures of cytochrome P4501B1. Am J Hum Genet 1998;62:573-584.
11. Colomb E, Kaplan J, Garchon HJ. Novel cytochrome P450 1B1 (CYP 1B1) mutations in patients with primary congenital glaucoma in France. Hum Mutat 2003;22: 496.

12. Reddy AB, Kaur K, Mandal AK, Panicker SG, et al. Mutation spectrum of the CYP1B1 gene in Indian primary congenital glaucoma. Mol Vis 2004;10:696-702.

13. Seligsohn U, Lubestsky A. Genetic subsceptibility to thrombosis. N Engl J Med 2001; 344:1222-1231.

14. Fainoi EM, Franchi F, Bucciarelli P, Margaglione M, et al. Coinheritance of the HR@ haplotype in the Factor $V$ gene confers an increased risk of venous thomboembolism to carriers of Factor V R506Q (Factor V Leiden). Blood 1999;94:3062-3066.

15. Strom CM. Mutation detection, interpretation and applications in the clinical laboratory setting. Mutat Res 2005;573:160-167. 